\title{
Treatment of Wounds from Fire Trench to Field Ambulance
}

\section{Commentary}

P Roberts

This is an account of one of the presentations at the meeting of the Royal Army Medical Corps, 3rd Corps Medical Society, in 1916. Reading so called 'classical papers' (in almost any subject) can invoke a host of reactions varying from outstandingly original, brilliant and worthy of a Nobel Prize, to fascinating but quaint! Short of a Nobel Prize, this paper does all of these and more. Any paper whose content ranges from producing improvised dressing sterilizers, the use of many-tailed bandages in the Franco Prussian War, the use of $10 \%$ saline solution and salt tablets as wound dressings to giving 2 pints of saline into the axilla in cases of shock, is both fascinating and quaint!

In the days when we attempt to deliver care that is evidence based, this approach may seem sadly lacking in this paper. It should be remembered that the management protocols eluded to are based on the best evidence available at the time albeit, not subject to much, if any, scientific scrutiny.

This paper gives a good flavour of problems faced by medical personnel, not only medical officers but also stretcherbearers, in attempting to alleviate suffering in the conditions that pertained in the trenches and the more rearward medical units. It talks about medical officers working under pressure who are not able to give time nor individual attention to each case. How true this is today. With this in mind, although implied, it is of interest that the word 'triage' does not appear at any time. Much is made of the requirement for medical officers to train their stretcher bearers to make treatment as efficient and appropriate as possible and that stretcher bearers should be men of intelligence who are interested in their work! They should not be selected because the are useless or physically incapable of regimental work. Do I see RMOs of today nodding in agreement when it comes to the selection of some
RMAs? Major Blackwood and his colleagues would have been very envious of today's equivalent of stretcher-bearers: the Combat Medical Technician.

There is much debate about the initial treatment of wounds, the control of haemorrhage and the management of shock. Do not touch the wound unnecessarily, cover it with a sterile dressing and do not disturb the dressing until something needs to be done with the wound. Despite the advent of anti-microbial agents, this is as true today as it was in the trenches in 1916.

Is the tourniquet an "invention of the Evil One?" The debate about the inappropriateness or otherwise of the use of tourniquets to control haemorrhage, rages just as much today as it did then. Major Blackwood contends that, if tourniquets are incorrectly applied or inappropriately used, loss of a limb will result; again how true today. But, he goes on to point out that practically all-external haemorrhage can be controlled by firm pressure with a pad over the bleeding point. Whether pads or first field dressings are impregnated with fibrin or not, again, some things do not and will not change!

The difficulties of casualty extraction, the requirement to keep casualties warm, the need for analgesia, the inappropriateness of primary wound closure, the need for adequate wound drainage, the requirement to modify surgical techniques when many casualties have to be dealt with, all of these things are in this fascinating paper.

This paper should be read against the background of Military Medical Science as it existed in 1916. It should then be re-read in the light of Military Medical Science in 2001. Clearly, major advances have been made but do not dismiss some of the apparently trivial points despite these medical advances. In a tight corner or austere situation, these trivia are just as important today as they were in the Great War!
Col P Roberts

QHS MBE MS FRCS

L/RAMC

Consultant Adviser in Surgery

AMD, Keogh Barracks, Ash Vale, Aldershot, Hants GU12 5RR 


\title{
RAMC Journal 1916 Vol 27 230-240 \\ ROYAL ARMY MEDICAL CORPS, 3rd CORPS MEDICAL SOCIETY TREATMENT OF WOUNDS FROM FIRE TRENCH T ${ }_{0}^{\circ}$ FIELD AMBULANCE
}

\author{
By Major Blackwood \\ Royal Army Medical Corps
}

This paper has been written, not with the idea of laying down any dogmatic rules for the treatment of wounded, but rather to focus attention on the various methods employed, and, by a statement of what our experience has been, to stimulate a discussion from which it is hoped we may all gain valuable information.

Let us commence with the treatment in the fire trench or aid post. I think it is advisable to consider it under two headings:

(a) In ordinary times.

(b) During a battle.

In the the first case, the wounded are few in number, and plenty of time is at the disposal of the medical officers for their treatment. In the second, the medical officers are working under pressure, probably not under the most ideal conditions for surgery, and therefore can neither give time nor individual attention to each case.

\section{At Ordinary Times in the Trenches}

In the large majority of cases the wounded will have received first-aid treatment from the regimental stretcher-bearers before being seen by the medical officer. Consequently, it devolves on the medical officer to train them so as to make their treatment as efficient as possible. The stretcher-bearers should be men of intelligence who are actually interested in their work, and on no account should they be men who have been selected because they are useless or physically incapable of regimental work. The work of the stretcher-bearer is very strenuous, and requires a man of strong physique.

There are three things in which the stretcher-bearer should be proficient: (a) The first-aid treatment of wounds; (b) the control of haemorrhage and the treatment of fractures; (c) the various methods for the removal of wounded from the trench to the aid post.

\section{First-aid Treatment of Wounds}

The stretcher-bearer is bound from his surroundings to be a most septic individual, and even with the best intentions in the world cannot render himself aseptic. Consequently, it should be impressed upon him that on no account is the wound to be touched. This fact must be well driven home; perhaps it might help if it was explained to him that his hands are covered with germs, and that if, by touching the wound, he should introduce only a single germ, that microb $\frac{\overline{\frac{5}{6}}}{\mathrm{~m}}$ will have the power of reproducing itself t? the extent of five or six millions in twenty four hours. This wonderful reproductive face is sure to strike the average "Tommy" Having got this fact impressed, the next thin is to instruct him how to approach th wound. This is a difficult problem, as wit many wounds it is necessary to remove th clothing, which will expose the patient to the danger of "cold", and in the case of a large wound may add considerably to the shock Personally, we feel that, under presen? conditions, where, with good communication trenches, it is possible at ale hours of the day to remove the patient to the aid post, as little exposure as possible in theo trench should take place.

Even with a well-fitted-out medical dugout in the trenches we do not think anys exposure is justified unless the medicalo officer is there and can personally carry out ao proper dressing. We see no reason why a을 small portion of the clothing cannot be cut $\overrightarrow{\overrightarrow{0}}$ away when it immediately surrounds the 3 wound, rather than undressing the patient, and a field or shell dressing applied; this would entail very little exposure and be far better than the indiscriminate cutting-up of the clothing which often happens now. A criss-cross cut made opposite the wound, and the flaps turned back, answers very well. Even in cases of severe bleeding, where a $\frac{5}{3}$ tourniquet has to be applied, complete exposure is not necessary, as the site of the wound is shown by the hole in the clothing, and further, the presence of the clothing will prevent the tourniquet bruising the skin. We also feel that full exposure of a wound means an added danger from further infection.

\section{Control of Haemorrhage}

We are inclined to think that tourniquets are an invention of the Evil One, and that it is no exaggeration to say that many limbs have been lost during this campaign by the indiscriminate use of them. We do not mean to say that, if a man is shot in the femoral or other large vessel, a tourniquet is not required, though here the majority of cases would be dead before a tourniquet could be found and applied. Our contention is that practically all external haemorrhage can be controlled by firm pressure with a pad over the bleeding point, at least until the field ambulance is reached, where it can be thoroughly dealt with. Such a pad can be 
made from a first field dressing or, if necessary, two field dressings, one on the top of the other. We know that there is the possibility of bleeding into the tissues, but this risk is small compared with the damage done by an unnecessary tourniquet. It should be remembered that, on the average, at least three or four hours must elapse before a case can be got from the fire trench to the field ambulance; and in that time a tourniquet may do irreparable damage. The Japanese method of attaching a strip of red flannel to cases wearing a tourniquet is worthy of notice. If, after applying these pads, the stretcher-bearer finds that considerable bleeding is still taking place, then he should use a tourniquet. We strongly advocate the issue of the round rubber tourniquet in place of the web tape with buckle, as it is more easily applied and more effective. A very simple and, at the same time, effective tourniquet can be improvised with a puttee and a piece of stick, the width of the puttee preventing any undue constriction. Where a limb is badly lacerated, the haemorrhage is seldom very considerable owing to twisting of the vessels, but the possibility of its occurrence should be watched for. The stretcher-bearer should also be instructed where to apply immediate digital pressure in case of injury to one of the large vessels.

\section{Fractures}

We think it advisable that the stretcherbearer should not apply splints to a fracture, especially of the lower limb, as it is very doubtful, when in the trenches, if he could apply them satisfactorily, and it would, besides, take a considerable time. We recommend that a fractured arm be bandaged against the trunk; and that, with fractures of the lower limb, the two feet be brought together and tied firmly, and again tied at the knees. In this way sufficient immobilizing of the limb will be obtained to move the wounded man to the aid post.

\section{Methods of Removal}

This now, with the present trenches, tramroads and communication trenches, is not difficult, as in practically all cases the wounded man can be carried on the ordinary stretcher. Cases may arise, however, where an ordinary stretcher cannot be used, where it may be necessary to use a Rogers's trench stretcher. This stretcher has not always found favour with the medical officers, but we think, with a little practice, the stretcherbearers will find the advantage of it in certain circumstances. The wounded should be removed to the aid post as soon as practicable after having been wrapped in blankets, it being remembered that any shock causes a rapid fall of temperature. Patients should be allowed to lie on the stretcher in the position they find most comfortable for breathing and ease, and not according to any hard and fast rules. Some sandbags might be kept filled with straw for use as pillows, which will add much to the comfort of the wounded man.

\section{At the Aid Post}

The medical officer should see all cases, and redress them except in the most trivial cases. It should be remembered that the first field dressing is an emergency dressing for application by the man himself or his companions, and was never intended to be looked upon as a permanent dressing. In wounds of the limbs, if tied tightly, it becomes a serious menace to the circulation. The stretcher-bearers should be specially warned of this, so that, in applying, he can make some allowance for this swelling.

Head cases should have the hair shaved off for at least two inches round the wound. It is not sufficient to clip it with scissors.

Chest and abdominal wounds should have a good pad of dressing applied over the wound, and retained in position by strapping; a bandage should never be passed round and round the body, as that entails additional movement for the patient. Protrusions of gut should be surrounded by lint wrung out in warm saline and covered with protective.

Fractures will require to be splinted. The question of extension does not arise at this stage - a good position is all that can be aimed at. A roll of Gooch's splinting will be found useful to cut splints from. A hypodermic injection of $1 / 2$ grain morphia will ease the pain during manipulation.

When a wound appears dirty and encrusted with mud, it should certainly be washed with 1 in 60 carbolic or 1 in 1,000 mercury lotion. The dressings should be kept in biscuit tins and handled only by the medical officer, who should try and carry out the ordinary rules of antiseptic surgery in respect of these dressings. The dressings should not be handled by the stretcherbearer unless he has been trained to carry out these rules. The dressings for wounds should be kept distinct from those used at the morning sick parade, where they are liable to be frequently handled by hands none too clean. The dressings most suitable are cyanide gauze with a plentiful supply of cotton-wool to prevent constriction by the bandage when swelling takes place. The surrounding skin should be well painted with four per cent iodine, which will destroy most skin organisms. If hairs are present on the surface they should be shaved off.

Treatment of shock is a point sometimes overlooked. It must be remembered that, after any wound, the temperature falls rapidly. Therefore the patient should be wrapped up in blankets. The boots should be removed from all stretcher cases, and loosened in that of walking patients, as this adds greatly to their comfort. Warm drinks or 
water should be given liberally, it being remembered that in all cases where haemorrhage has occurred the system calls out for water. This is only contra-indicated in abdominal wounds, where it should only be given in sips, and under the orders of the medical officer. Chest and abdomen cases should be warned against any sudden movements, either by the patients or attendants.

Morphia may be given freely in all cases, and in order to get the maximum result, it is advisable to give it hypodermically. We have seen many cases where it has been given by the mouth without any appreciable result. It is especially indicated in wounds of the abdomen and thorax, as it tends to keep the patient quiet, and so prevents movement. We also think the use of morphia helps to tide over the period of shock. It can be given in much larger doses than is generally taught without any ill effect. All cases that have had much haemorrhage should be made to lie down with the head low, and on no account to sit up. Brandy may be given in cases with much collapse, both for its mental and physical effects.

\section{In Battle Times}

Here the conditions are entirely altered, as the numbers of wounded have made it practically impossible for the medical officer to give individual attention to all the cases, his attention necessarily being called to the most serious. The exactness of surgical technique has more or less to give way to speed, in order that the cases may be got away to the field ambulance as speedily as possible, both for the benefit of the wounded and to prevent chokage in the front line. The suggestions already given for treatment should be carried out as closely as possible. It will be found that probably first field and shell dressings will have to be used more frequently than in the "quiet time", and therefore it is again necessary to draw attention to the danger of tieing the tapes on these dressings too tightly, as it is quite probable that delays may occur, preventing the case getting to the field ambulance for some considerable time. The same warning will equally apply to tourniquets. Fractures should be immediately immobilized in splints before removal. Rolls of Gooch's splinting will be found most useful, as splints of varying size can be cut as required from the roll, which besides, is compact and easily carried. A bottle of liquid morphia hypodermica should be carried by the medical officer, as being more handy for use than the tablet, and far more effective than oral administration. Water should be given freely to the wounded, and clothing cut up as little as possible. The wounded should be encouraged to walk whenever possible, in order that the ambulance and regimental stretcher-bearers may be used for the carriage of serious cases.

The Field Ambulance - The role played by the field ambulance in modern warfare is that of a "drainage company", which aims at $\frac{\pi}{\mathbb{\alpha}}$ counteracting, as far as possible, the $\varrho$ inevitable sepsis of all wounds. It was this "inevitable spesis" which took us some time to realize, in the early part of the War, trained as we all were in the aseptic school. Soon it $\stackrel{\vec{F}}{+}$ was found that all sutures closing wounds을 simply prevented the escape of pus, and $\frac{\bar{\sigma}}{\overline{0}}$ experience showed that the more open all wounds were left the better were the results obtained. Hence the duty of the ambulance is to open rather than close the wound, and $\overrightarrow{0}$ so provide for the free escape of all $\overrightarrow{\vec{\omega}}$ discharges. This drainage of wounds should $\stackrel{\omega}{S}$ be carried out in a thorough manner, otherwise it is useless, and we may be excused, therefore, if we go into a little fully, $\frac{\vec{A}}{\vec{A}}$ as we feel it is of the greatest importance for $\vec{v}$ the future recovery of our wounded and the $N$ shortening of convalescence - both points of primary importance to an army in the field. 음 In January of last year we started using $\vec{c}$ drainage tube of the diameter of $1 / 2$ inch to $\subseteq$ one inch, and have continued doing so ever $\stackrel{\widehat{D}}{\mathrm{D}}$ since, the drainage tube as found in the $\tilde{O}$ surgical panniers being too small to be of $\stackrel{-}{-}$ much use. Small and superficial wounds and wounds of muscle with small points of entry and exit with no injury to bone do not require drainage, or at least should be given the benefit of the doubt, as they frequently $\stackrel{\unrhd}{\unrhd}$ arrive at the base quite clean when dressed $\overrightarrow{\overrightarrow{0}}$ with a four per cent iodine solution and dry 3 sterilized gauze. In wounds, however, of any size, and where a bone is involved, drainage must be free. Frequently we have found, with only a small wound of entry and exit, but where the bone has been injured, that on opening up there is a large cavity inside. Drainage, to be of any use, must be free, so $\bar{F}$ in the majority of cases requiring drainage it $\frac{\sigma}{3}$ is necessary to give an anaesthetic. Counteropening may be required, in the making of $\frac{0}{3}$ which the future position of the limb in 0 subsequent dressings must be considered, and, if possible, they should be made through the most dependent point. The counteropenings should be large enough to admit $\sigma$ the drainage tube easily. A tube should never $N$ be passed diagonally across a fractured limb, స్ల so as to traverse a defect in the bone or to lie in close proximity to any large vessel. The $\stackrel{0}{工}$ tubing should have numerous lateral openings cut in it, or be slit right up, and if it be slightly pointed at one end and the bullet forceps found in the operation case be passed $\stackrel{\mathbb{D}}{\Omega}$ through the wound tract and used to grasp $\stackrel{\mathbb{Q}}{\Omega}$ this pointed end, no difficulty will be found in introducing the largest size tubing. Care $\delta$ should be taken to insert a sterilized safety pin into the end of the tubing to prevent it slipping in, and the tubing should be cut off fairly short to prevent the end being closed by the pressure of the dressings. It is better to 
err on the side of over-doing rather than under-doing the drainage, free drainage being the one preventive of gas gangrene. In some cases it is better to cut through any small bridges of skin and muscle, so as to allow of subsequent free granulating. Occasionally, wounded come in who have laid out for some time after the receipt of their injury, and who may show symptoms of gas gangrene in varying degrees. This condition is easily recognized by the smell and by a dirty brown fluid which escapes from the wound, and it requires to be very freely opened up, both by incisions into the muscles and by drainage tubes.

Anaesthetics - They should be given in the majority of wounds of any size or severity, and experience has shown that they are required in about ten per cent of all wounds, otherwise it is quite impossible to carry out a thorough cleansing and drainage of the wound. Open ether is the ideal anaesthetic, both from the point of view of immediate safety and from the subsequently depressing effects of chloroform. No difficulty will be found in producing complete narcosis with a little practice. One-hundredth of a grain of atropine, with or without morphia, should be given if possible beforehand.

Dressings - They should be all sterilized. We have been fortunately situated in having a steam sterilizer, but one can be easily improvised from a biscuit tin. We have lately used a method of sterilizing of dressings for minor wounds which we find most excellent, as it avoids soiling a large amount of dressings; while, for the large wounds, we use dressings from the drum of the sterilizer. We also use this sterilizer for towels and operating gowns.

The wound we wash with warm water and ether soap, then bathe in a lotion of either 1 in 1,000 mercury or 1 in 60 carbolic, followed by ether, and finally swab as dry as possible before painting with four per cent iodine. In no case should iodine be applied to large raw surfaces, as it is useless owing to the moisture present in these cases. The iodine should always, however, be painted on to a wide area round the wound. Cyanide gauze is then applied. If a large raw surface has to be dressed, we find that gauze soaked in equal parts of percxide of hydrogen and glycerine is good, as it will prevent the gauze from sticking when redressed. Plugging of wounds should be avoided, as it interferes with the drainage, and it must be very occasionally that it is necessary for stopping haemorrhage in that manner. The bleeding points should be cut down on and ligatured, as they are generally easy to find. We use boiled silk for all ligatures. Gloves should be worn, both as a protection to the patient and to the operator's hands from the effects of the antiseptic lotions. Portions of projectile we leave, unless easy of removal or a cause of discomfort to the patient.
In serious head injuries we shave the head, an operation which should never be done unless the patient is under an anaesthetic. The question of the necessity of an immediate trephine to relieve grave pressure symptoms must be left to the experience of the surgeon. We had excellent results last winter from cases treated by us, as at that time we were allowed to retain them for ten or twelve days.

Fractures we treat on the general lines laid down. No fragments of bone should be removed unless absolutely free and of small size.

There are several splints that we find most useful:

(1) Page's arm and femur splint.

(2) Our leg splint.

(3) Thigh splint with interruption.

Shock and Collapse - All cases show this in varying degrees, and the treatment runs on more or less general lines. When admitted, the patient should have any wet clothing removed, boots taken off, and dry socks put on. Bed boots made of flannel or other warm material, and reaching to just below the knee, will be found useful. He should be well wrapped up in blankets, and with hot-water bottles if necessary.

Warm drinks should be given - patients prefer cocoa or tea to bovril. Water should not be stinted if asked for. We find morphia an excellent line of treatment, as it composes the patient and he drops off to sleep. Morphia is especially useful in wounds of the chest and pneumothorax, which seem to produce great mental shock. If collapse is severe, especially if due to haemorrhage, we find elevation of the feet, the use of pituitrin and subcutaneous or intravenous injections of normal saline give excellent results. With a medium-sized needle from the Potair aspirating case and a funnel and tube, a couple of pints can be quickly run into the axilla or thighs. Continuous saline by the

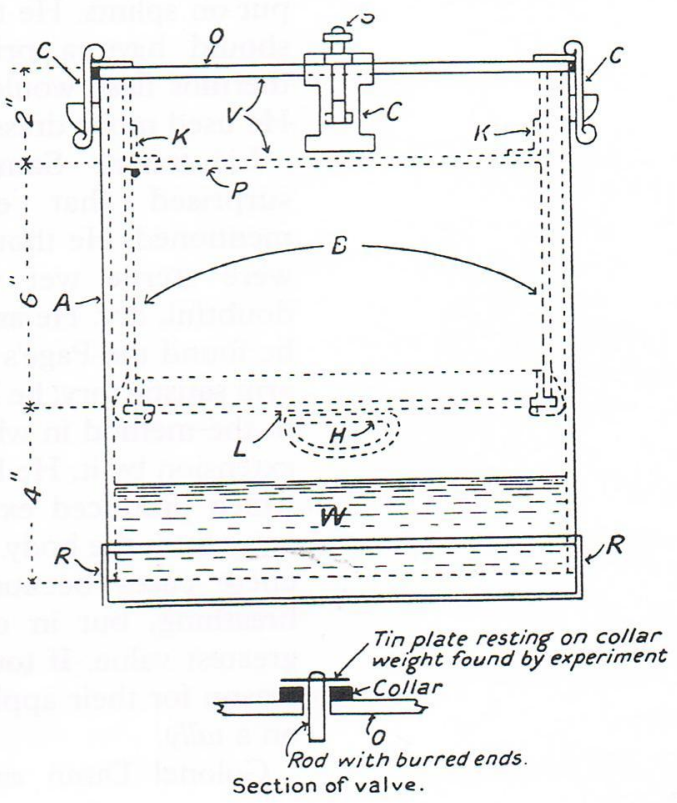


changes, redressings, and, therefore, not so suitable in a field ambulance.

Colonel Bruce Skinner remembered that after the Franco-Prussion War every surgeon was going to do away with the ordinary bandage for splints and adopt the manytailed, and McCormick after the RussoTurkish War said the same. He had used strips of bandage over splints as a student, and he still considered it better than using a whole one. He read the introduction to Sir Almroth Wright's "Memorandum on the Employment of Vaccines in connexion with Wound Infections". The wound could be packed with salt tablets and not dressed again until the base is reached, and as the flow is from the wound to the surface it is difficult to see how infection of the wound can take place. He wished to bring attention to:

(1) The training of stretcher-bearers; they must be repeatedly taught, not only a series of lectures and then finish.

(2) The very ingenious sterilizer exhibited, pointing to the desire felt for sterilizers in a field ambulance.

(3) The bed-socks exhibited were presented by the Corps Commander.

(5) Primus stoves had been allotted for some time to aid posts. 